\title{
Effect of power and rate adaptation on the spectral efficiency of MQAM/OFDM system under very fast fading channels
}

\author{
Zhicheng Dong ${ }^{1,2^{*}}$, Pingzhi Fan ${ }^{1}$, Erdal Panayirci ${ }^{3}$ and P Takis Mathiopoulos ${ }^{4}$
}

\begin{abstract}
In this article, the effect of power and rate adaptation on the spectral efficiency of orthogonal frequency division multiplexing (OFDM) systems using M-ary quadrature amplitude modulation (MQAM) is investigated in the presence of frequency selective and very rapidly time-varying fading channels, under power and instantaneous bit error rate (BER) constraints. Lower bounds on the maximum spectral efficiency of adaptive OFDM/MQAM systems with perfect and imperfect channel state information (CSI) are obtained, together with a closed-form expression for the average spectral efficiency of adaptive OFDM systems. The delay between the channel estimation and the actual transmission is also considered in this article. The theoretical and numerical results show that the adaptive MQAM/OFDM systems under fast fading channels have substantial gains in spectral efficiency over the non-adaptive counterparts with perfect CSI or moderate imperfect CSI. The theoretical and numerical results also show that a good way to deal with the delay CSI under very rapidly time-varying channels is to increase the subcarrier bandwidth of the OFDM system while ignoring the impact of the cyclic prefix.
\end{abstract}

Keywords: OFDM, Intercarrier interference (ICI), Power and rate adaptation, Spectral efficiency, High mobility

\section{Introduction}

Orthogonal frequency-division multiplexing (OFDM) has been shown to be an effective technique to overcome the inter-symbol interference (ISI) caused by frequencyselective fading with a simple transceiver structure. It has emerged as the leading transmission technique for a wide range of wireless communication standards [1] such as the IEEE's 802.16 family-better known as Mobile Worldwide Interoperability Microwave Systems for Next-Generation Wireless Communication Systems (WiMAX)-and the Third-Generation Partnership Project (3GPP) in the form of its long-term evolution (LTE) project. Both systems employ orthogonal frequency division multiplexing/multiple access (OFDMA) as well as a new singlecarrier frequency-division multiple access (SC-FDMA) format. To promote the IEEE 802.16 and LTE standards, recently, a high mobility feature has been introduced

\footnotetext{
*Correspondence: dongzc666@163.com

${ }^{1}$ Institute of Mobile Communications, Southwest Jiaotong University,

Chengdu, 610031, China

${ }^{2}$ School of Engineering, Tibet University, Lhasa, 850000, China

Full list of author information is available at the end of the article
}

(IEEE 802.16m, LTE Advanced (LTE-A)) to enable mobile broadband services at vehicular speeds beyond $120 \mathrm{~km} / \mathrm{h}$.

In fading channels with very high mobilities, the time variation of the channel over an OFDM symbol period results in a loss of subchannel orthogonality which leads to inter-channel interference (ICI) due to power leakage among OFDM subcarriers making the system more sensitive to fast fading channels. ICI caused by the time selectivity of wireless channel will degrade the spectral efficiency of the system [2-5]. To maximize the spectral efficiency of the system, power and bit loading algorithms have been derived to adaptively adjust power and data rates across subcarriers according to the instantaneous channel state information (CSI) at the transmitter [6]. However, it is impossible to obtain perfect channel information at the transmitter due to channel estimation error and feedback delay. Many researchers have studied the performance and have proposed possible solutions under imperfect CSI at the transmitter. Bit and power loading algorithms were pursued in [7-14], where partial CSI was utilized to adapt the constellation size and/or the power, adhering to a certain target bit error rate (BER) 
per subcarrier. However, the authors did not consider the effect of ICI.

Some articles studied OFDM systems under carrier frequency offset (CFO) [15-19]. The CFO also results in ICI and destroys orthogonality among the subcarriers. Cheon and Hong [15] derived the average SNR and BER of OFDM systems in the presence of CFO. Rugini and Banelli [16] derived the BER of OFDM systems with CFO in frequency-selective Rician and Rayleigh fading channels. Based on [15], Nehra and Shikh-Bahaei presented the spectral efficiency of adaptive M-ary quadrature amplitude modulation (MQAM)/OFDM systems with CFO over fading channels [17]. In [17], the upper bounds of the OFDM under CFO is also presented. But this scheme cannot meet the BER constraint for every subcarrier. In [18], Das et al. proposed adaptive bit loading in conjunction with an adaptive subcarrier bandwidth. However, adaptive power distribution and imperfect CSI were not considered. In [19], Wen et al. designed an optimal joint bit loading and power allocation scheme for OFDM systems in the presence of ICI. However, the optimal solution must be achieved by an iterative method, and the imperfect CSI was not considered.

In order to study adaptive MQAM/OFDM systems, one should investigate first the forms of the SNR and BER expressions in the presence of very rapidly time varying channels. Much of the research on the performance analysis of OFDM in fast fading channels are based on an approximation of the ICI components as Gaussian random variables or by using the average ICI power. Such simple closed-form expressions and bounds for the total ICI power are presented in [11,20-22]. These have been employed to obtain approximate expressions for the average signal-to-interference plus noise ratio (SINR) [23-25], and error rates [3-5].

The main objective of this article is to consider extending OFDM with adaptive modulation to very rapidly time varying channel environments. The effect of the ICI due to the fast fading channel on the spectral efficiency of the power and rate adapted OFDM systems is investigated. Using MQAM with perfect and imperfect CSI at the transmitter, we formulate the bit and power allocation problem and derive new analytical expressions for optimal rate and power adaptation to maximize the spectral efficiency while satisfying an average power constraint and instantaneous BER target in the presence of the fast fading channels. The second major role of this work is to investigate how the imperfections in CSI, due to the channel estimation errors as well as the delay between channel estimation and the actual transmission, impact the system performance and how we can improve the performance, under again very rapidly time varying channel conditions. Note that many articles have achieved the channel estimation by estimating the time-varying channel impulse response by using a pilot symbol assisted technique $[26,27]$. However, the channel estimation errors in the time domain result in ICI in the frequency domain. We analyze the effect of the imperfect CSI on the spectral efficiency, under average power and instantaneous BER constraints. In particular, we derive closed form expressions for the achievable maximum capacity of the system with fast fading channel from which several important conclusions are reached concerning the system design.

The rest of this article is organized as follows: System model section introduces an OFDM based system model, the time-varying wireless channel and the ICI caused by the nature of the channel. Non-adapitve MQAM/OFDM scheme section discusses the nonadaptive MQAM/OFDM scheme with fast fading channels. The average spectral efficiency as well as the maximum average capacity expressions of the adaptive MQAM/OFDM are derived in Adaptive MQAM/OFDM scheme section with perfect channel information. In Spectral efficiency of adaptive OFDM with imperfect CSI section, the effects of both the channel estimation error and the outdated CSI are investigated. Finally, in Numerical results and discussion section, the simulation and numerical results are presented along with some concluding remarks.

\section{System model}

We consider an OFDM system with $N$ subcarriers. The time-domain transmitted signal can be written as

$$
s(n)=\frac{1}{\sqrt{N}} \sum_{k=0}^{N-1} d_{k} e^{j 2 \pi n k / N},-L \leq n \leq N-1
$$

where $L$ is the length of the guard interval and $d_{k}$ stands for the frequency domain data symbol transmitted over the $k$ th OFDM subchannel. We assume that all symbols have the same energy $E_{s}=E\left\{\left|d_{k}\right|^{2}\right\}$ per subcarrier, Then the received signal at the input of the FFT takes the known form

$$
y(n)=\sum_{l=0}^{L} h(n, l) s(n-l)+w(n)
$$

where $h(n, l)$ is the channel impulse response of the $l$ th tap at time $n$. We assume Rayleigh fading channels, and a Jakes' Doppler spectrum with maximum Doppler frequency $f_{\max }=f_{c} v / c$, where $f_{c}$ is the carrier frequency $(\mathrm{Hz}), v$ is the speed of the mobile terminal $(\mathrm{km} / \mathrm{h})$, and $c$ is the speed of light. Assume that the maximum channel delay spread is less than or equal to the guard interval $L$, and $w(n)$ is zero-mean, complex additive white Gaussian noise with variance $\sigma_{w}^{2}$. The $k$ th subcarrier output from the FFT can be expressed as [26] 


$$
\begin{aligned}
Y_{k} & =\frac{1}{\sqrt{N}} \sum_{n=0}^{N-1} y(n) e^{-j 2 \pi n k / N} \\
& =d_{k} H_{k}+I_{k}+W_{k}
\end{aligned}
$$

where

$$
\begin{aligned}
H_{k} & =(1 / N) \sum_{n=0}^{N-1} H_{k}(n) \\
I_{k} & =1 / N \sum_{m=0, m \neq k}^{N-1} d_{m} \sum_{n=0}^{N-1} H_{m}(n) e^{j 2 \pi n(m-k) / N} .
\end{aligned}
$$

$H_{k}(n)$ in (4) is the Fourier transform of the timevarying channel impulse response at time $n$, defined as $H_{k}(n)=\sum_{l=0}^{L-1} h(n, l) \exp (-j 2 \pi l k / N)$ and $W_{k}=\frac{1}{\sqrt{N}}$ $\sum_{n=0}^{N-1} w(n) \exp (-j 2 \pi n k / N)$. The variance of $W_{k}$ is $\sigma_{w}^{2}$. Note that $H_{k}(n) \sim \mathcal{C N}(0,1)$ and $E\left\{H_{k}(n) H_{k}^{*}\left(n^{\prime}\right)\right\}=$ $J_{0}\left(2 \pi f_{\max } T_{\text {OFDM }}\left(n-n^{\prime}\right) / N\right)$, where $T_{\text {OFDM }}=1 / \Delta f=$ $N / B$, and $B$ is the total bandwidth $(\mathrm{Hz})$ (we assume the overhead due to the guard interval is ignored in this article, but the guard interval would reduce the spectral efficiency in practical OFDM systems). $f_{\max } T_{\text {OFDM }}=$ $f_{c} v N / c B$ is the normalized Doppler frequency of the system. $J_{0}($.) denotes the zeroth-order Bessel function of the first kind.

The second term in (3) represents intercarrier interference (ICI) caused by the time-varying nature of the channel. From (5), the ICI power of the $k$ th OFDM subcarrier can be obtained as

$$
P_{\mathrm{ICI}}^{(k)}=E\left\{\left|I_{k}\right|^{2}\right\}=\sum_{m=0, m \neq k}^{N-1} E\left\{\left|d_{m}\right|^{2}\right\} \rho_{k, m}
$$

where

$$
\begin{aligned}
\rho_{k, m}= & \frac{1}{N^{2}}\left(N+2 \sum_{n=1}^{N-1}(N-n) J_{0}\left(2 \pi f_{\max } T_{\mathrm{OFDM}} n / N\right)\right. \\
& \times \cos (2 \pi n(k-m) / N)) .
\end{aligned}
$$

Since the data on each subcarrier are uncorrelated, and $E\left\{\left|d_{m}\right|^{2}\right\}=E_{s}$, it follows easily from (6) that the ICI power, $P_{\mathrm{ICI}}^{k}$, is independent of the subcarrier index $k$ and has the following form $[20,26]$ :

$$
P_{\mathrm{ICI}}=E_{s}\left(1-\frac{1}{N^{2}}\left(N+2 \sum_{n=1}^{N-1}(N-n) J_{0}\left(2 \pi f_{\max } T_{\mathrm{OFDM}} n / N\right)\right)\right) \text {. }
$$

To simplify the issue, the normalized ICI power $P_{N}$ is adopted in this article, where $P_{N}=P_{\mathrm{ICI}} / E_{s}$. When $f_{c}, N$, and $B$ are constant, $P_{\mathrm{ICI}}$ increases with the speed $v$. Note that, in a time-invariant channel, the value of $v$ is zero therefor $P_{\mathrm{ICI}}$ becomes also zero. In line with the channel model of $[10,17]$, we make two assumptions:

(1) Each subchannel is narrow enough that it experiences frequency-flat fading.

(2) $H_{k}$ has a complex Gaussian distribution with zero mean and variance $\rho_{0}$ for all $k$ at each OFDM interval [26], where

$$
\begin{aligned}
\rho_{0}= & E\left\{\left|H_{k}\right|^{2}\right\}=\left(N+2 \sum_{n=1}^{N-1}(N-n) J_{0}\right. \\
& \left.\times\left(2 \pi f_{\max } T_{\text {OFDM }} n / N\right)\right) / N^{2} .
\end{aligned}
$$

Assuming that the transmitted symbols are mutually uncorrelated, the instantaneous effective SINR for the $k$ th subcarrier with fixed power allocation can be written as [15],

$$
\operatorname{SINR}_{k}=\left|H_{k}\right|^{2} \bar{\gamma} /\left(P_{N} \bar{\gamma}+1\right)=\gamma[k] /\left(P_{N} \bar{\gamma}+1\right)
$$

where $|H|_{k}^{2} \bar{\gamma}=\gamma[k]$ is the instantaneous SINR for the $k$ th subcarrier and $\bar{\gamma}$ is the average SNR when there is no ICI. The average effective SINR is then defined as

$$
\overline{\operatorname{SINR}}_{k}=\frac{E\left\{\left|H_{k}\right|^{2}\right\} \bar{\gamma}}{P_{N} \bar{\gamma}+1}=\frac{\bar{\gamma} \rho_{0}}{P_{N} \bar{\gamma}+1} .
$$

From (10), it follows that $\overline{\operatorname{SINR}}_{k}$ decreases as the speed increases when $\bar{\gamma}$ is fixed. Figure 1 shows the average effective SINR as a fuction of $\bar{\gamma}$. As can be seen from Figure 1, the received average SINR increases with the average transmitted SNR. When the velocity equals $100 \mathrm{~km} / \mathrm{h}$, it changes almost linearly as function of the average SNR. However, when the velocity reaches $500 \mathrm{~km} / \mathrm{h}$, the trend of the increase in the SINR is no longer linear due to the fact that the ICI also increases with speed.

\section{Non-adapitve MQAM/OFDM scheme}

We now consider, as a first case, a non-adaptive modulation scheme where $\beta(\gamma[k])=\beta$ is a constant for all $k$. Assuming $M$-ary QAM symbols are transmitted over each subchannel of an OFDM system, the number of bits/symbol sent over the $k$ th subchannel is $\beta(\gamma[k])=$ $\log _{2} M(\gamma[k])$. The instantaneous BER for the $k$ th subcarrier is approximated by [28]:

$$
\begin{aligned}
\Omega([k]) \approx & \frac{2}{\beta(\gamma[k])}\left(1-\frac{1}{\sqrt{2^{\beta(\gamma[k])}}}\right) \operatorname{erfc} \\
& \times\left(\sqrt{1.5 \frac{\gamma[k]}{\left(2^{\beta(\gamma[k])}-1\right)\left(P_{N} \bar{\gamma}+1\right)}}\right)
\end{aligned}
$$




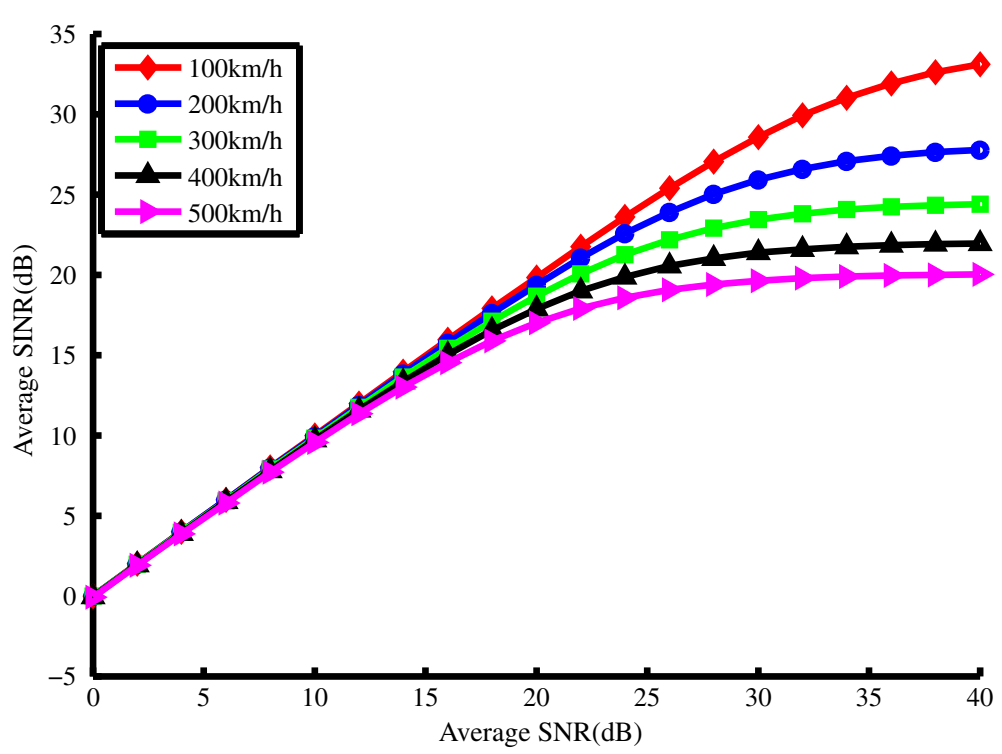

Figure 1 Average SINR versus average SNR.

Since (11) can not be easily invertible, we consider a different BER approximation formula with these properties [28].

$$
\Omega([k]) \approx C_{1} \exp \left(\frac{-C_{2} \gamma[k]}{\left(2^{\beta(\gamma[k])}-1\right)\left(P_{N} \bar{\gamma}+1\right)}\right)
$$

where $C_{1}=0.2, C_{2}=1.6$.

Assuming that the subchannel $H_{k}$ is a complex Gaussian random variable and all $H_{k}$ have identical distributions with $E\left\{\left|H_{k}\right|^{2}\right\}$ given by (8), the average BER can be approximated as

$$
\begin{aligned}
\Omega^{\prime} & \approx \int_{0}^{\infty} C_{1} \exp \left(\frac{-C_{2} v \bar{\gamma}}{\left(2^{\beta}-1\right)\left(P_{N} \bar{\gamma}+1\right)}\right) p_{v}(v) d v \\
& =C_{1}\left(\frac{C_{2} \rho_{0} \bar{\gamma}}{\left(2^{\beta}-1\right)\left(P_{N} \bar{\gamma}+1\right)}+1\right)^{-1}
\end{aligned}
$$

where $p_{v}(v)=1 / \rho_{0} \exp \left(-v / \rho_{0}\right)$ and $\rho_{0}$ is defined by (8).

Assuming $\varepsilon$ is the target average BER, the maximum bit load, given the average BER constraint is

$$
\beta=\log _{2}\left[\frac{C_{2} \rho_{0} \bar{\gamma}}{\left(C_{1} / \varepsilon-1\right)\left(P_{N} \bar{\gamma}+1\right)}+1\right]
$$

Figure 2 shows the tightness of the simplified average BER approximation for MQAM, according to (12), with different bit rates for velocities 100 and $500 \mathrm{~km} / \mathrm{h}$, respectively. It is evident that the simplified approximation nearly predicts the BER expression of (11). It also shows that the BER performance degrades with an increase in vehicle speed when the bit rate is fixed. In order to guarantee BER performance, the bit rate must be reduced under high mobility.

\section{Methods}

\section{Adaptive MQAM/OFDM scheme}

For adaptive OFDM, different power levels and modulation bit load are allocated to different subchannels. The instantaneous BER of each subchannel is

$$
\Omega([k]) \approx C_{1} \exp \left(\frac{-C_{2} \gamma[k] \frac{s(\gamma[k])}{\bar{S}}}{\left(2^{\beta(\gamma[k])}-1\right)\left(P_{N}^{(k)} \bar{\gamma}+1\right)}\right)
$$

where the transmit power $s(\gamma[k])$ and $\beta(\gamma[k])$ are adapted with $\gamma[k]$, subject to the average power $\bar{S}$, and the instantaneous BER constraints $\Omega([k])=\varepsilon . \quad P_{N}^{(k)}$ is the normalized ICI power (variance) of the $k$ th subcarrier. From (6) it follows that

$$
P_{N}^{(k)}=\sum_{m=0, m \neq k} \frac{s(\gamma[k])}{\bar{s}} \rho_{k, m}
$$

Note that it is very difficult to determine the exact $P_{N \_ \text {ICI }}^{(k)}$ when all the transmit powers carried by the subcarriers are not the same. However, we can obtain an upper bound in terms of the largest transmit normalized power $s_{\max }=$ $\max _{m}\{s(\gamma[m]) / \bar{S}\}, \sum_{m=0}^{N-1} s(\gamma[m]) / \bar{S}=1$, for all the subcarriers, as will be explained shortly. Consequently, employing the upper bound on the normalized ICI variance of all subcarriers, determined by $s_{\max }$, the upper bound of the instantaneous BER for each subchannel can be expressed as

$$
\Omega([k]) \leq C_{1} \exp \left[\frac{-C_{2} \gamma[k] \frac{s(\gamma[k])}{\bar{s}}}{\left(2^{\beta(\gamma[k])}-1\right)\left(s_{\max } P_{N} \bar{\gamma}+1\right)}\right]
$$




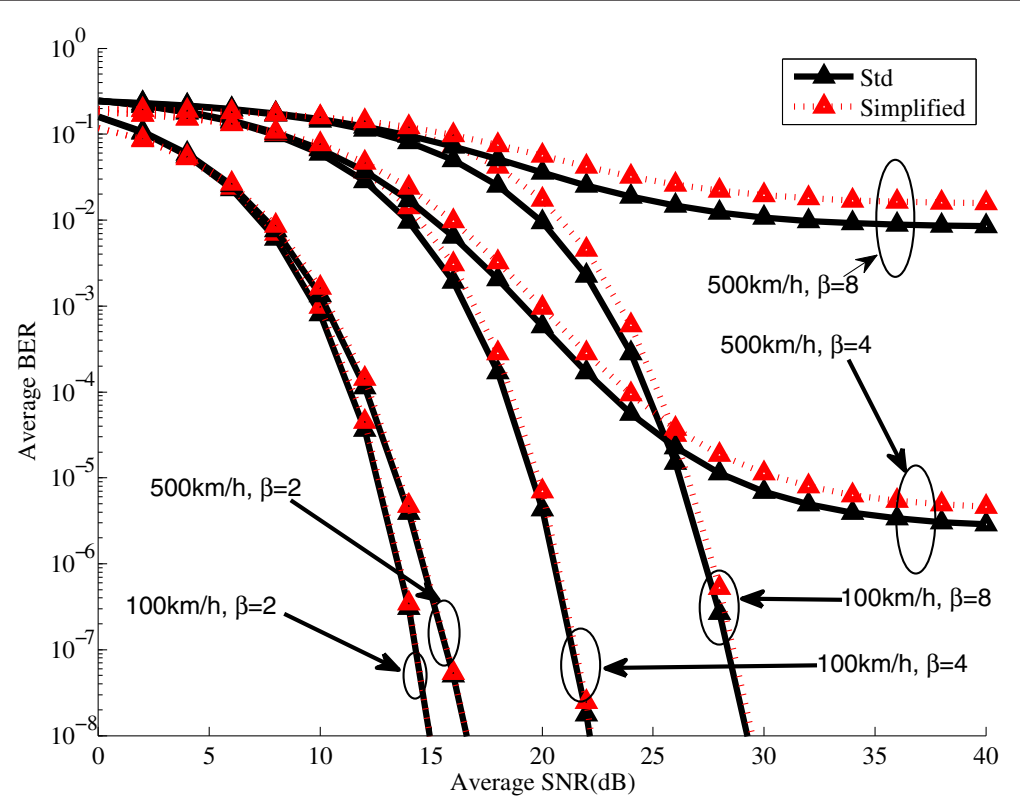

Figure 2 Average BER versus average SNR.

where $P_{N}$ is the normalized ICI power when all the subchannels carry the same average power, determined from (6) as $P_{N}=\sum_{m=0, m \neq k}^{N-1} \rho_{k, m}$. According to (17), the maximum bit load size for each subchannel is obtained as

$$
\beta(\gamma[k]) \leq \log _{2}\left[1+\frac{\left(-C_{2} / \ln \left(\varepsilon / C_{1}\right)\right) \gamma[k] \frac{s([k])}{\bar{s}}}{\left(s_{\max } P_{N} \bar{\gamma}+1\right)}\right] .
$$

The maximum bit load in (18) is a lower bound for each subchannel because of the largest ICI power. According to Assumption 2 for the channel model, it can be shown that the average capacity of an adaptive OFDM system does not depend on the system parameters or the specific power delay profile of the wide sense stationary uncorrelated scattering (WSSUS) mobile channels [17].

To maximize the average capacity of an adaptive OFDM system, the following constrained optimization problem is considered next:

$$
\max _{s(\gamma[k])} \int \beta(\gamma[k]) p_{\gamma[k]}(\gamma[k]) d \gamma[k]
$$

subject to

$$
\begin{array}{lr}
E_{\gamma[k]}\{s(\gamma[k])\} \leq \bar{S} & \forall k \in N \\
s(\gamma[k]) \geq 0 & \forall k \in N \\
\Omega([k]) \leq \varepsilon & \forall k \in N
\end{array}
$$

where $E_{x}\{\cdot\}$ denotes expectation with respect to $x$. Then, the Lagrangian for the optimization problem can be defined as

$$
\begin{aligned}
J\{s(\gamma[k])\}= & \int \log _{2}\left[1+\frac{\left(-C_{2} / \ln \left(\varepsilon / C_{1}\right)\right) \gamma[k] \frac{s(\gamma[k])}{\bar{S}}}{\left(s_{\max } P_{N} \bar{\gamma}+1\right)}\right] \\
& \times p_{\gamma[k]}(\gamma[k]) d \gamma[k]-\lambda \\
& \times\left(\int s(\gamma[k]) p_{\gamma[k]}(\gamma[k]) d \gamma[k]-\bar{S}\right)
\end{aligned}
$$

where $\lambda$ is the Lagrange multiplier. The optimal power adaptation must be nonnegative and satisfy

$$
\frac{\partial J\{s(\gamma[k])\}}{\partial s(\gamma[k])}=0 \quad s(\gamma[k]) \geq 0 \quad \beta(\gamma[k]) \geq 0 .
$$

Solving (21) yields the optimal power adaptation

$$
\frac{s(\gamma[k])}{\bar{S}}=\frac{1}{\lambda \ln (2) \bar{S}}-\frac{s_{\max } P_{N} \bar{\gamma}+1}{\left(-C_{2} / \ln \left(\varepsilon / C_{1}\right)\right) \gamma[k]}
$$

where the value of the Lagrange multiplier $\lambda$ can be obtained from the average power constraint using numerical methods. The optimal rate adaptation is then given as

$$
\beta(\gamma[k])=\log _{2}\left[\frac{\left(-C_{2} / \ln \left(\varepsilon / C_{1}\right)\right) \gamma[k]}{(\lambda \ln (2) \bar{S})\left(s_{\max } P_{N} \bar{\gamma}+1\right)}\right] \text { bps } / \mathrm{Hz} .
$$


In practice, however, only an integer value of the rate is acceptable. Therefore, the rate is truncated to the nearest integer value.

The maximum average capacity of an adaptive OFDM system is determined by averaging $\beta(\gamma[k])$ over the probability distribution of $\gamma[k]$. Consequently, it is computed from

$$
\mathrm{Cap}_{\max }=\int_{\gamma 0}^{\infty} \log _{2}\left[\frac{\left(-C_{2} / \ln \left(\varepsilon / C_{1}\right)\right) \gamma[k]}{(\lambda \ln (2) \bar{S})\left(s_{\max } P_{N} \bar{\gamma}+1\right)}\right] p_{\gamma[k]}(\gamma[k]) d \gamma[k]
$$

where $\gamma_{0}=\frac{\left(s_{\max } P_{N} \bar{\gamma}+1\right) \lambda \ln (2) \bar{S}}{\left(-C_{2} / \ln \left(\varepsilon / C_{1}\right)\right)}$ is the optimized threshold for $\gamma[k]$ below which the channel is not used. Note that this threshold can be found from (22) so that $\frac{s(\gamma[k])}{\bar{S}} \geq 0$. To find a closed-form expression for (24), we use the pdf of $p_{\gamma[k]}(\gamma[k])$ given by

$$
p_{\gamma[k]}(\gamma[k])=\frac{\exp (-\gamma[k] / \overline{\gamma[k]})}{\overline{\gamma[k]}}
$$

were, $\overline{\gamma[k]}=\bar{\gamma} E\left\{\left|H_{k}\right|^{2}\right\}$ is the average received SNR when there is no ICI. As can be seen from (8), $E\left\{\left|H_{k}\right|^{2}\right\}$ is independent of the subcarrier index $k$. Therefore, $\overline{\gamma[k]}$ can be substituted by $\rho_{0} \bar{\gamma}$. As shown in Appendix 1, (24) can be evaluated as

$$
\operatorname{Cap}_{\max }=-\frac{1}{\ln (2)} E i\left(-\frac{\gamma_{0}}{\rho_{0} \bar{\gamma}}\right)
$$

where $E i(\cdot)$ denotes the exponential integral [29].

In order to evaluate $s_{\max }$ in (20), we employ the instantaneous BER expression (15) for each subchannel $k$ where the normalized ICI power for that subchannel is computed. However, the resulting constrained optimization problem stated by (19a)-(19d) to find the optimal average capacity of the $k$ th subchannel in an adaptive OFDM system needs the knowledge of the other subchannel powers, $s(\gamma[m]) / \bar{S}, m=0,1, \ldots, N-1(m \neq k)$ which makes the solution computationally intractable. One feasible way to solve the problem is to assume that the normalized ICI power of the $k$ th subchannel is determined by its own power, that is, $P_{N_{-} \text {ICI }}^{(k)} \approx s(\gamma[k]) / \bar{S} \times P_{N}$. However, the BER performance, in this case, cannot meet the constraint (19d) since the transmit powers of the adjacent subcarriers are larger than that of the $k$ th subcarrier. The fact that $H_{k}$ being the best subchannel implies that $s(\gamma[k]) / \bar{S}$ as well as $P_{N_{-} \text {ICI }}^{(k)}$, determined by $s(\gamma[k]) / \bar{S}$, take their maximum values resulting in the constraint (19d) satisfied. Consequently, $s_{\max }$ can be determined as $s_{\max }=\lim _{\gamma[k] \rightarrow \infty} s(\gamma[k]) / \bar{S}$. We can obtain an upper bound from (22) as

$$
s_{\max }=\lim _{\gamma[k] \rightarrow \infty}\left(\frac{s(\gamma[k])}{\bar{S}}\right)=\frac{1}{\lambda \ln (2) \bar{S}}
$$

For the worst case, the ICI is determined by the largest power, when $\gamma[k]=\infty$ and a lower bound on the maximum spectral efficiency in adaptive OFDM/MQAM systems is obtained.

\section{Spectral efficiency of adaptive OFDM with imperfect CSI}

In practice, it is impossible to obtain perfect channel information due to noisy channel estimation and the unavoidable delay between when channel estimation is achieved and when the estimation result is used for actual transmission. We will consider imperfect CSI in this section and study the performance degradation in spectral efficiency resulting from these CSI errors.

\section{Effect of imperfect CSI}

In a practical system, the channel estimator at the receiver provides the channel gains estimates $H_{k}^{\prime}(n)$, which are assumed as $H_{k}^{\prime}(n)=H_{k}(n)+\epsilon_{k}(n)$, where $\epsilon_{k}(n)$ represents the channel estimation error that is independent of the true channel gain $H_{k}(n)$ and is distributed according to $\mathcal{C N}\left(0, \sigma_{\epsilon}^{2}\right)$ [30]. Consequently, the distribution of $H_{k}^{\prime}=(1 / N) \sum_{n=0}^{N-1} H_{k}^{\prime}(n)$ becomes $\mathcal{C N}\left(0, \rho_{0}+\sigma_{\epsilon}^{2} / N\right)$ where $\rho_{0}=E\left\{\left|H_{k}\right|^{2}\right\}$ and is given by (8). Suppose the estimated channel gain $H_{k}^{\prime}$ is the only known information about the current CSI for the $k$ th subcarrier. Since the instantaneous BER of each subcarrier, $\Omega([k])$, depends on the value of the true channel $H_{k}$, through $\gamma^{\prime}[k]=$ $\bar{\gamma}\left|H_{k}^{\prime}\right|^{2}$ which is assumed unknown, it is not possible to fix $\Omega^{\prime}$ ( $[k]$ ) to be the target value. However, we can define the average BER given $H_{k}^{\prime}$ for the $k$ th tone, as explained below.

As shown in Appendix 2, the instantaneous BER of each subchannel under imperfect CSI can be expressed as

$$
\Omega^{\prime}([k]) \leq C_{1} \exp \left[\frac{-C_{2} \gamma[k] \frac{s\left(\gamma^{\prime}[k]\right)}{\bar{s}}}{\left(2^{\beta\left(\gamma^{\prime}[k]\right)}-1\right) \phi}\right]
$$

where $\phi=s_{\max }^{\prime} P_{N} \bar{\gamma}+s_{\max }^{\prime} \bar{\gamma}(N-1) \sigma_{\varepsilon}^{2} / N+1$.

Consider the special case when $H_{k}$ given $H_{k}^{\prime}$ is complex Gaussian with mean $\mu$ and variance $\sigma^{2}$. It follows that $z=\left|H_{k}\right|$ conditioned on $H_{k}^{\prime}$ has a Ricean distribution [10]. Then the average BER for the $k$ th subcarrier is defined as $\bar{\Omega}([k])=E_{z \mid H_{k}^{\prime}}\{\Omega([k])\}$. Taking the expectation 
over the Ricean distribution, the average BER can be expressed as

$$
\begin{aligned}
\bar{\Omega}([k])= & \int_{0}^{\infty} C_{1} \exp \left[\frac{-C_{2} z^{2} \bar{\gamma} \frac{s\left(\gamma^{\prime}[k]\right)}{\bar{s}}}{\left(2^{\beta\left(\gamma^{\prime}[k]\right)}-1\right) \phi}\right] \frac{2 z}{\sigma^{2}} \\
& \times \exp \left\{-\frac{z^{2}+|\mu|^{2}}{\sigma^{2}}\right\} I_{0}\left(\frac{2 z|\mu|}{\sigma^{2}}\right) d z
\end{aligned}
$$

where $I_{0}(\cdot)$ is the zeroth-order modified Bessel function of the first kind. Taking the integral in (29), we obtain the the average BER:

$$
\bar{\Omega}([k])=C_{1} f\left(\gamma^{\prime}[k]\right) \exp \left(-\frac{|\mu|^{2}}{\sigma^{2}}\left[1-f\left(\gamma^{\prime}[k]\right)\right]\right)
$$

where $f\left(\gamma^{\prime}[k]\right)$ is defined as

$$
f\left(\gamma^{\prime}[k]\right)=\left(1+\frac{C_{2} s\left(\gamma^{\prime}[k]\right) \bar{\gamma} \sigma^{2}}{\bar{S}\left(2^{\beta\left(\gamma^{\prime}[k]\right)}-1\right) \phi}\right)^{-1} .
$$

Let $f\left(\gamma^{\prime}[k]\right)$ be the appropriate function in (30) such that it will set an upper bound $\varepsilon$ for the instantaneous BER, that is, $\bar{\Omega}([k]) \leq \varepsilon$. Consequently, from (30) we have

$$
C_{1} f\left(\gamma^{\prime}[k]\right) \exp \left(-\frac{|\mu|^{2}}{\sigma^{2}}\left[1-f\left(\gamma^{\prime}[k]\right)\right]\right)=\varepsilon
$$

Note that in (30), $f($.) cannot be solved in a closed mathematical form. However, $f\left(\gamma^{\prime}[k]\right)$ can be approximated by $\varphi\left(\gamma^{\prime}[k]\right)[31]$ :

$$
\varphi\left(\gamma^{\prime}[k]\right)=1+\frac{\sigma^{2}}{|\mu|^{2}} \log \left(\varepsilon / C_{1}\right)
$$

Closeness of the approximation $\varphi\left(\gamma^{\prime}[k]\right)$ to $f\left(\gamma^{\prime}[k]\right)$ is shown in Figure 3, over the entire range of $|\mu|^{2} / \sigma^{2}$.

The following relation between the power and rate of the $k$ th subcarrier can be derived from (31)

$$
\beta\left(\gamma^{\prime}[k]\right)=\log _{2}\left[\frac{s\left(\gamma^{\prime}[k]\right) C_{2} \bar{\gamma} \sigma^{2}}{\bar{S} \phi\left(\frac{1}{f\left(\gamma^{\prime}[k]\right)}-1\right)}+1\right] .
$$

To solve the optimization problem for power adaptation, its Lagrangian for the optimization problem is defined as

$$
\begin{aligned}
J\left\{s\left(\gamma^{\prime}[k]\right)\right\}= & \int \log _{2}\left[\frac{s\left(\gamma^{\prime}[k]\right) C_{2} \bar{\gamma} \sigma^{2}}{\bar{S} \phi\left(\frac{1}{f\left(\gamma^{\prime}[k]\right)}-1\right)}+1\right] p_{\gamma^{\prime}[k]} \\
& \times\left(\gamma^{\prime}[k]\right) d \gamma^{\prime}[k]+\lambda_{1}\left(\int s\left(\gamma^{\prime}[k]\right) p_{\gamma^{\prime}[k]}\right. \\
& \left.\times\left(\gamma^{\prime}[k]\right) d \gamma^{\prime}[k]-\bar{S}\right) .
\end{aligned}
$$

The value of Lagrangian multiplier $\lambda_{1}$ can be found from the average power constraints by using numerical methods, subject to the same constrained optimization problem presented in (19b). Following the same analytical derivations as that of (22), the optimal power adaptation is obtained as

$$
\frac{s\left(\gamma^{\prime}[k]\right)}{\bar{S}}=-\frac{1}{\lambda_{1} \ln (2) \bar{S}}-\frac{\phi\left(\frac{1}{f\left(\gamma^{\prime}[k]\right)}-1\right)}{C_{2} \bar{\gamma} \sigma^{2}},
$$

from which the corresponding optimal rate adaptation is found as

$$
\beta\left(\gamma^{\prime}[k]\right)=\log _{2}\left[-\frac{C_{2} \bar{\gamma} \sigma^{2}}{\phi\left(\lambda_{1} \ln (2) \bar{S}\right)\left(\frac{1}{f\left(\gamma^{\prime}[k]\right)}-1\right)}\right] .
$$

Finally, the maximum average spectral efficiency can be expressed as

$$
\begin{aligned}
C^{\prime} \operatorname{ap}_{\max }= & \int_{\gamma_{01}^{\prime}}^{\infty} \log _{2}\left[-\frac{C_{2} \bar{\gamma} \sigma^{2}}{\phi\left(\lambda_{1} \ln (2) \bar{S}\right)\left(\frac{1}{f\left(\gamma^{\prime}[k]\right)}-1\right)}\right] \\
& \times p_{\gamma^{\prime}[k]}\left(\gamma^{\prime}[k]\right) d \gamma^{\prime}[k]
\end{aligned}
$$

where $\gamma_{01}^{\prime}$ is an optimized threshold below which the channel is not employed. It can be determined from

$$
f_{\varepsilon}\left(\gamma_{01}^{\prime}\right)=\left[1-\frac{C_{2} \bar{\gamma} \sigma^{2}}{\lambda_{1} \ln (2) \bar{S} \phi}\right]^{-1}
$$

where $f_{\varepsilon}()=.f($.$) which satisfies (32).$ 


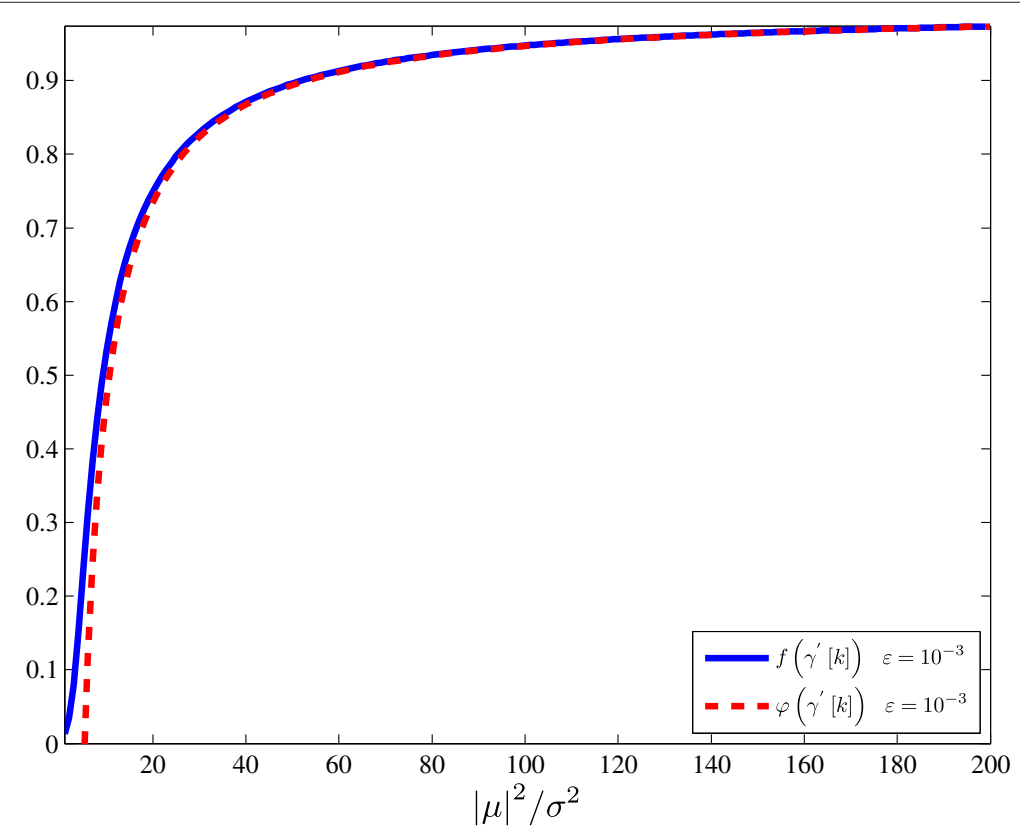

Figure $3 f\left(\gamma^{\prime}[k]\right)$ and $\varphi\left(\gamma^{\prime}[k]\right)$ as a function of $|\mu|^{2} / \sigma^{2}$.

Using the Gaussian error assumption, it can be shown that $H_{k}$ given $H_{k}^{\prime}$ is complex Gaussian with mean and variance given by [10],

$$
\mu_{1}=\frac{\rho_{0}}{\rho_{0}+\sigma_{\epsilon}^{2} / N} H_{k}^{\prime}, \quad \sigma_{1}^{2}=\frac{\rho_{0}\left(\sigma_{\epsilon}^{2} / N\right)}{\rho_{0}+\sigma_{\epsilon}^{2} / N} .
$$

Replacing $\mu$ and $\sigma^{2}$ in (38) with $\mu_{1}$ and $\sigma_{1}^{2}$, respectively, we can obtain the average spectral efficiency,

$$
\begin{aligned}
C^{\prime} \operatorname{ap}_{\max }= & \int_{\gamma_{01}^{\prime}}^{\infty} \log _{2}\left[-\frac{C_{2} \bar{\gamma} \sigma^{2}}{\phi\left(\lambda_{1} \ln (2) \bar{S}\right)\left(\frac{1}{f\left(\gamma^{\prime}[k]\right)}-1\right)}\right] \\
& \times p_{\gamma^{\prime}[k]}\left(\gamma^{\prime}[k]\right) d \gamma^{\prime}[k] \\
= & -\frac{1}{\ln (2)}\left(\frac{\varepsilon}{C_{1}}\right)^{\frac{\sigma_{\varepsilon}^{2}\left(\rho_{0} N+\sigma_{\varepsilon}^{2}\right)}{\rho_{0}^{2} N^{2}}} E i\left(\varpi^{\prime}\right)
\end{aligned}
$$

where $\varpi^{\prime}=-\lambda_{1} \ln (2) \phi \ln \left(\frac{\varepsilon}{C_{1}}\right)\left(\rho_{0}^{2} N^{2}+2 \rho_{0} N \sigma_{\varepsilon}^{2}+\sigma_{\varepsilon}^{4}\right) /$ $\left(N^{2} \rho_{0}^{3} C_{2}\right)$.

Evaluation of $s_{\max }^{\prime}$ in (28) is similar to the derivations for the evaluations of $s_{\max }$ presented in Adaptive MQAM/OFDM scheme section. For the worst case, the ICI is decided by the largest power. We can obtain the largest power if $\gamma^{\prime}[k] \rightarrow \infty$. A lower bound on the maximum spectral efficiency in adaptive OFDM/MQAM systems is then obtained as

$$
s_{\max }^{\prime}=\lim _{\gamma^{\prime}[k] \rightarrow \infty}\left(\frac{s\left(\gamma^{\prime}[k]\right)}{\bar{S}}\right)=-\frac{1}{\lambda_{1} \ln (2) \bar{S}}
$$

\section{Delay in CSI}

We now investigate the performance degradation in power and rate adaptation caused by the delay transmitting CSI from the receiver to the transmitter. We assume perfect CSI. However, the channel estimate at the transmitter due to the transmission delay is expressed as $H_{k}^{\prime}(n)=H_{k}(n-\Delta n)$, where $\tau_{D}=\Delta n T_{\text {OFDM }}$ is the time delay, in seconds, between the time at which the channel estimation is performed and the actual transmission. It follows from (5) that the correlation between $H_{k}(n)$ and $H_{k}^{\prime}(k)$ is

$$
r=E\left\{H_{k}^{\dagger} H_{k}^{\prime}\right\}=\frac{1}{N^{2}} \sum_{n=0}^{N-1} \sum_{n^{\prime}=0}^{N-1} E\left\{H_{k}^{\dagger}(n) H_{k}^{\prime}(n)\right\}
$$

Based on the assumption that the channel has a WSSUS model, it can be shown that [26]

$$
E\left\{H_{k}^{\dagger}(n) H_{k}^{\prime}(n)\right\}=J_{0}\left(\frac{2 \pi f_{\max } T_{\text {OFDM }}\left(n-n^{\prime}+\Delta n\right)}{N}\right) .
$$


Then, (42) can be expressed as

$$
\begin{aligned}
r= & \frac{1}{N^{2}}\left(N J_{0}\left(\frac{2 \pi f_{\max } T_{\text {OFDM }} \Delta n}{N}\right)+\sum_{n=1}^{N-1}(N-n) J_{0}\right. \\
& \times\left(\frac{2 \pi f_{\max } T_{\text {OFDM }}(n+\Delta n)}{N}\right)+\sum_{n=1}^{N-1}(N-n) J_{0} \\
& \left.\times\left(\frac{2 \pi f_{\max } T_{\text {OFDM }}(n-\Delta n)}{N}\right)\right) .
\end{aligned}
$$

Since $H_{k}$ and $H_{k}^{\prime} \sim \mathcal{C N}\left(0, \rho_{0}\right)$, it follows from (4) that the conditional distribution of $H_{k}$ given $H_{k}^{\prime}$ is complex Gaussian with mean and variance $\mu_{2}=\left(r / \rho_{0}\right) H_{k}^{\prime}$ and $\sigma_{2}^{2}=\rho_{0}-r^{2} / \rho_{0}$, respectively. Similar to the previous derivations, the average BER $\bar{\Omega}$ ([ $k]$ ) for the $k$ th subchannel can be determined from (32) with $\mu=\mu_{2}$ and $\sigma^{2}=$ $\sigma_{2}^{2}$. The instantaneous BER of each subchannel is

$$
\Omega^{\prime}([k]) \leq C_{1} \exp \left[\frac{-C_{2} \gamma^{\prime}[k] \frac{s\left(\gamma^{\prime}[k]\right)}{\bar{s}}}{\left(2^{\beta\left(\gamma^{\prime}[k]\right)}-1\right)\left(s_{\max }^{\prime \prime} P_{N} \bar{\gamma}+1\right)}\right]
$$

Going over the same steps starting from (28), as previously done for noisy channel estimation, we can obtain the maximum spectral efficiency as

$$
C^{\prime \prime} \operatorname{ap}_{\max }=-\frac{1}{\ln (2)}\left(\frac{\varepsilon}{C_{1}}\right)^{\frac{\rho_{0}^{2}-r^{2}}{r^{2}}} E i\left(\frac{\ln \left(\frac{\varepsilon}{C_{1}}\right)\left(\rho_{0} P_{N}-\rho_{0} \lambda_{2} \ln (2)\right)}{C_{2} r^{2}}\right)
$$

where $\lambda_{2}$ is a Lagrangian multiplier and its value is determined from the average power constraint.

The evaluation of $s_{\max }^{\prime \prime}$ in (44) is similar to the derivations for the evaluations of $s_{\max }$ presented in Adaptive MQAM/OFDM scheme section. For the worst case, the ICI is decided by the largest power. We can obtain the largest power if $\gamma^{\prime}[k] \rightarrow \infty$. A lower bound on the maximum spectral efficiency in adaptive OFDM/MQAM systems is then obtained,

$$
s^{\prime \prime}{ }_{\max }=\lim _{\gamma^{\prime}[k] \rightarrow \infty}\left(\frac{s\left(\gamma^{\prime}[k]\right)}{\bar{S}}\right)=-\frac{1}{\lambda_{2} \ln (2) \bar{S}}
$$

\section{Numerical results and discussion}

In this section, we examine the performance of the solution for the power and rate adaptation derived in the previous sections. In all simulations presented here, we assume the total bandwidth $B$ is $15.36 \mathrm{MHz}$, the numbers of subcarriers are $N=1024, N=512$ or $N=$ 256, and the corresponding subcarrier bandwidths are $\Delta f=15 \mathrm{KHz}, \Delta f=30 \mathrm{KHz}$ or $\Delta f=60 \mathrm{KHz}, f_{c}=$ $2.5 \mathrm{GHz}, v=100$ and $500 \mathrm{~km} / \mathrm{h}$. The BER requirement is $10^{-3}$. In Figure 4 , the adaptive power control scheme $s(\gamma[k]) / \bar{S}$ is plotted as a function of the average SNR. The figure shows similar trends with respect to $\gamma[k]$ for different mobilities. It also indicates that higher speeds yield larger cutoffs and transmit powers as $\gamma[k] \rightarrow+\infty$ mainly due to fact that the ICI power also gets larger. Higher transmit power at higher speeds as $\gamma[k] \rightarrow+\infty$ is also due to the average power constraint.

In Figure 5, the bit rate adaptation $\beta(\gamma[k])$ is plotted as a function of the average SNR. We conclude from Figure 5

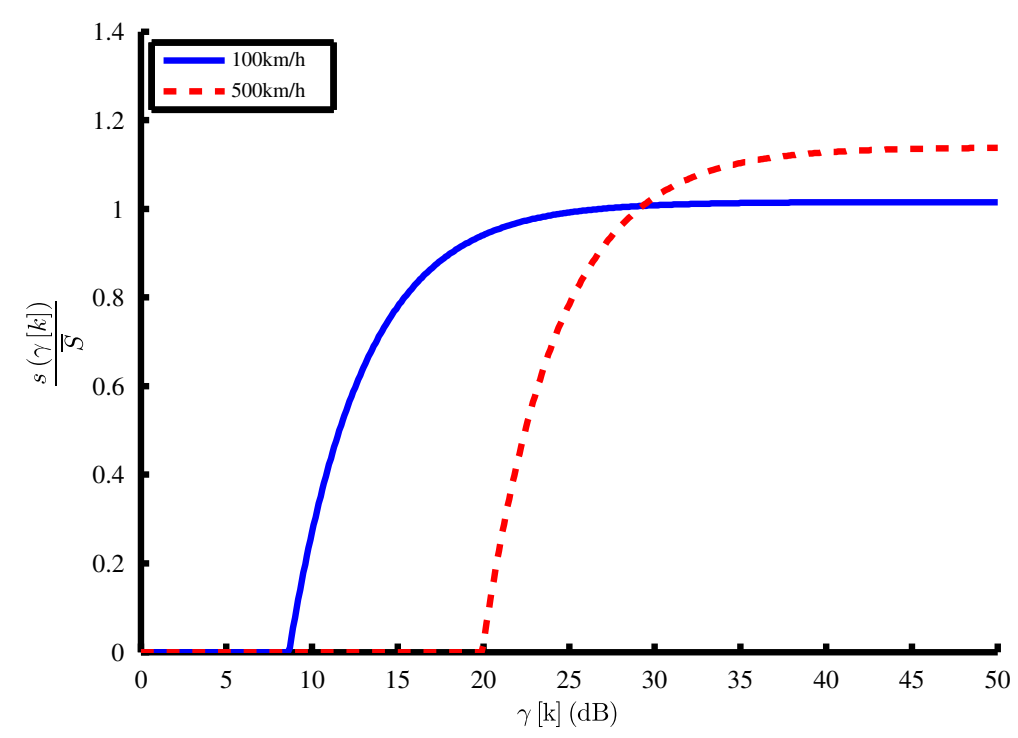

Figure $4 \frac{s(\gamma[k])}{\bar{s}}$ for MQAM $\left(\overline{\mathrm{BER}}=10^{-3}, \overline{\gamma[k]}=35 \mathrm{~dB}\right)$. 


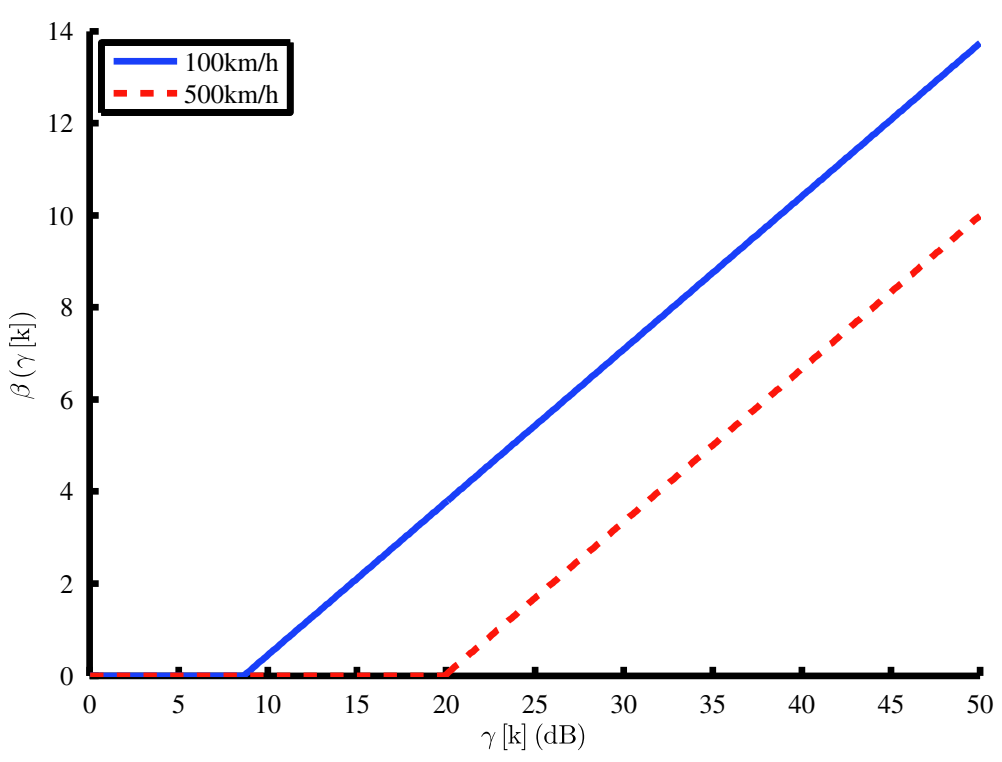

Figure $5 \beta(\gamma[k])$ for MQAM $\left(\overline{\mathrm{BER}}=10^{-3}, \overline{\gamma[k]}=35 \mathrm{~dB}\right)$.

that it is possible to transmit more bits per second as $\gamma[k]$ increases and that the higher speed yields the larger cutoff. It also shows that the lower speed has a larger bit rate than its higher speed counterpart when $\gamma[k]$ is fixed. As can be seen in Figure 4, the higher speed case yields a lower bit rate than that for the lower speed case, even with a larger transmit power, because of the larger ICI variance obtained at higher speeds.

Figure 6 shows the average spectral efficiency of adaptive OFDM systems as well as of non-adaptive OFDM for different speeds. It also indicates that a higher speed result in lower average spectral efficiency, mainly due to the fact that it induces larger ICI power. As can be seen in Figure 6 the adaptive OFDM scheme can achieve higher gains in spectral efficiency compared with non-adaptive OFDM under different velocities. However, they both experience error floors at high SNR values in the average spectral efficiency. We also observed that the spectral efficiency of the system cannot be improved beyond a certain level; this is mainly due to the contribution of the average SNR to the ICI power. The average spectral efficiencies of truncated integer rate adaptive OFDM are also given in Figure 6. In spite of the fact that its spectral efficiency is close to that of adaptive OFDM, we observe that there is still a gap between them, since the optimal parameters are obtained in the rate-domain.

The performance of adaptive OFDM systems with imperfect channel estimation at speeds 100 and $500 \mathrm{~km} / \mathrm{h}$ are shown in Figures 7 and 8, respectively. The value of $\sigma_{\varepsilon}^{2}$ is a measure of the quality of the channel estimation technique employed. The smaller the value, the better the channel estimation; $\sigma_{\varepsilon}^{2}=-\infty$ indicates that we have perfect CSI. From Figures 7 and 8, we can conclude that as the channel estimation becomes poor, the average spectral efficiency decreases, almost independently from the user mobility. The fact that the effect of noise resulting from imperfect CSI will be dominant compared to the ICI effect. For example, when $\sigma_{\varepsilon}^{2}=-20 \mathrm{~dB}$, the performance is worse than the non-adaptive for some values of SNR, as seen from Figure 7. However, Figure 8 shows that the performance loss is moderate when the channel estimation error is less than $-40 \mathrm{~dB}$. For the same channel estimation error, the performance loss incurred for low speed systems are greater than for high speed systems, since the low speed systems rely on more accurate CSI to improve the system performance. Consequently, we conclude that good channel stimulation is very essential in adaptive modulation schemes to improve the average spectral efficiency performance. The results also show that the closed-form expressions for adaptive OFDM systems with imperfect channel estimation is valid.

There is no impact of delay on the non-adaptive systems. However, for adaptive schemes, an unavoidable delay occurs between the times at which the channel estimation takes place and when the estimation result is employed for actual transmission. It is very important and necessary to investigate this issue, especially for high mobility systems. In Figure 9, we assume $N=1024, f_{c}=$ $2.5 \mathrm{GHz}, \Delta f=15 \mathrm{KHz}, v=100$ and $500 \mathrm{~km} / \mathrm{h}$, the delay $\tau_{D}=1 / \Delta f$ means a duration of one OFDM symbol. In this article, we assume that the overhead due to the guard interval is ignored by simply assuming the cyclic prefix $(\mathrm{CP})$ is equal to zero. Figure 9 shows that the higher the velocity, the worse the performance. Because the higher 


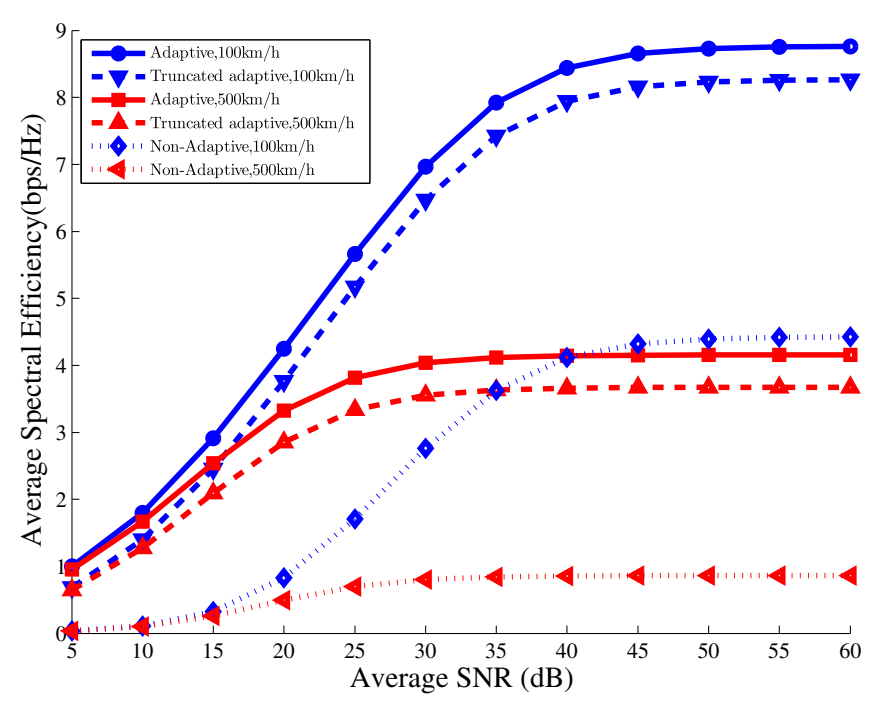

Figure 6 Average spectral efficiency versus average SNR.

mobility results in a larger variation of the channel within one OFDM symbol duration, the higher mobility results in decreasing channel correlation. We observe from Figure 9 that the adaptive scheme operating with a velocity equal to $100 \mathrm{~km} / \mathrm{h}$, it can resist the same delay (one OFDM duration) better than system with a velocity of $500 \mathrm{~km} / \mathrm{h}$. Consequently, under the high mobility condition, in order to mitigate the impact of delay, an effective and simple scheme is to reduce the OFDM symbol duration if the maximum path delay is not large.

Figure 10 shows the average spectral efficiency of an adaptive OFDM system with different delays and with different numbers of subcarriers. The velocity is chosen to be $500 \mathrm{~km} / \mathrm{h}$. From this figure we conclude that in order to mitigate the impact of delay, we need to increase the OFDM subcarrier spacing when the total bandwidth $B$ is constant. We assume the $\mathrm{CP}$ is ignored. Clearly, for a fixed total bandwidth $B$, the OFDM symbol duration with $N=512$ is half of that when $N=1024$ and consequently, the impact of delay would be reduced when $N=512$. Figure 10 shows that the OFDM system with $N=256$ is less affected by the delay. Meanwhile, increasing the subcarrier spacing of the OFDM system will also reduce the ICI effect when the velocity is constant. In practice, if

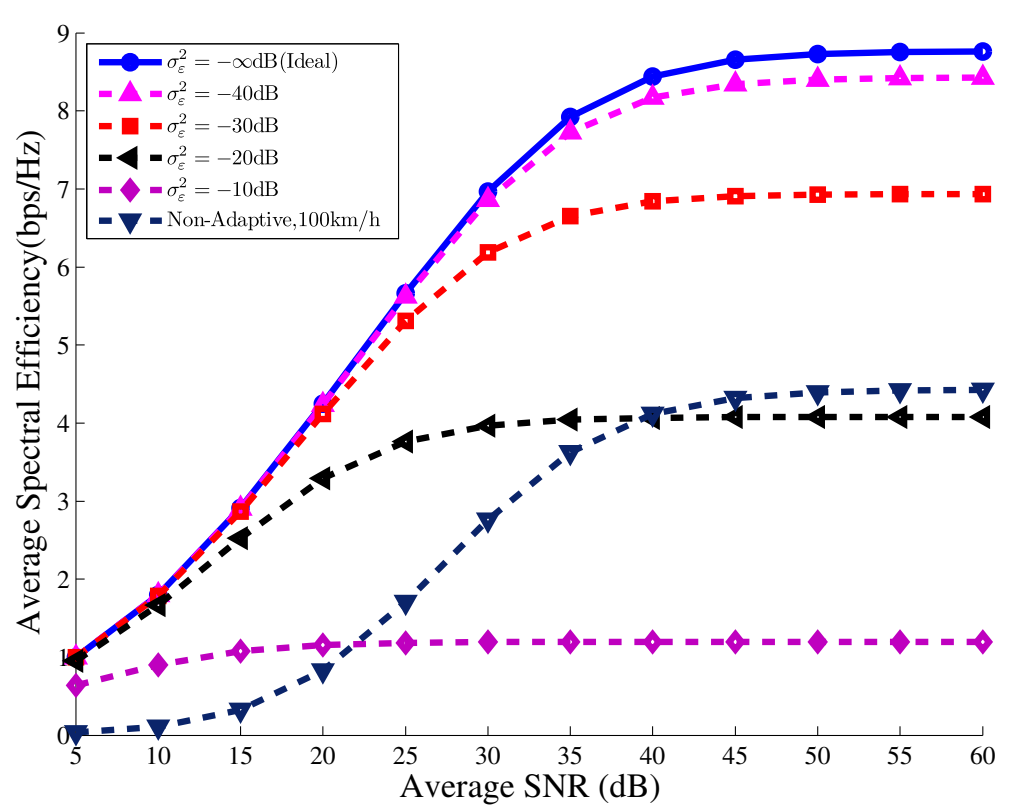

Figure 7 Average spectral efficiency for adaptive OFDM with imperfect CSI for velocity equals to $100 \mathrm{~km} / \mathrm{h}$. 


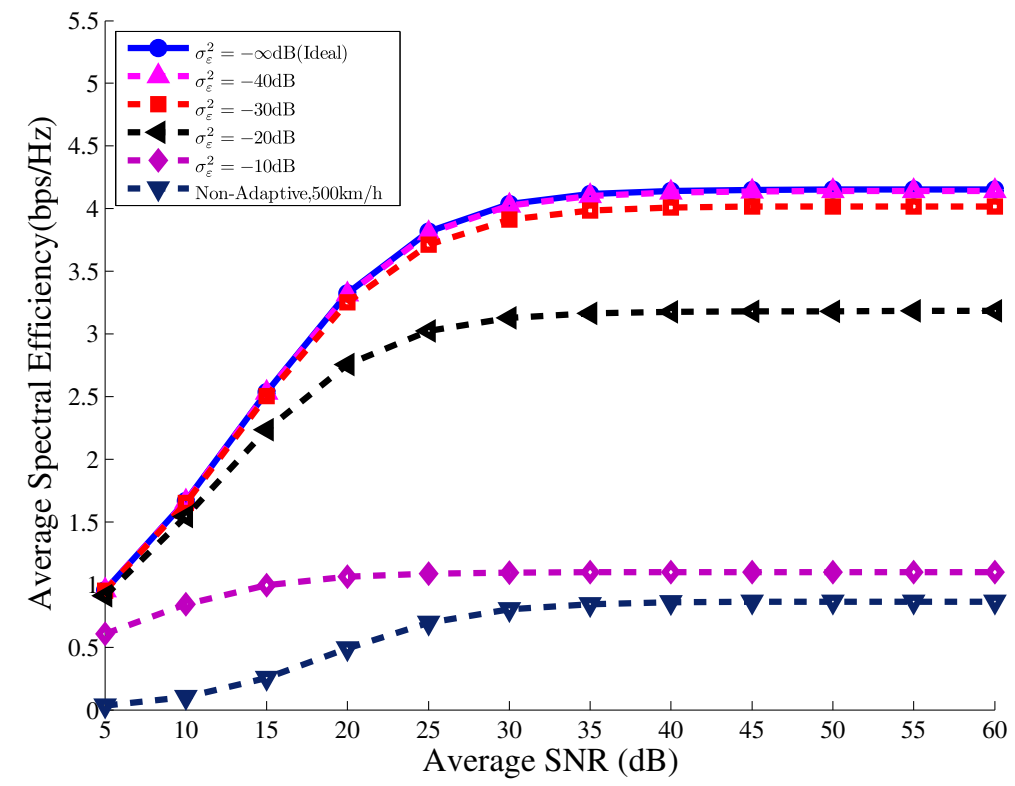

Figure 8 Average spectral efficiency for adaptive OFDM with imperfect CSI for velocity equals to $500 \mathrm{~km} / \mathrm{h}$.

the subcarrier spacing of an OFDM system is increased, the average spectral efficiency would be reduced when the maximum path delay is constant. So there is a tradeoff.

\section{Conclusion}

In this article, a power and rate adaptation scheme has been studied to maximize average spectral efficiency of MQAM/OFDM systems in a fast fading channel with perfect and imperfect CSI. Especially when dealing with high mobility and imperfect CSI, the banded structure of the channel matrix in the frequency domain has been taken into account. Under imperfect CSI, the effect of channel estimation errors and the delay in CSI on the spectral efficiency of the system were investigated. In order to exploit the impact of the ICI due to high mobility, new BER expressions were obtained for adaptive modulation in the presence of fast fading channels with perfect and imperfect CSI.

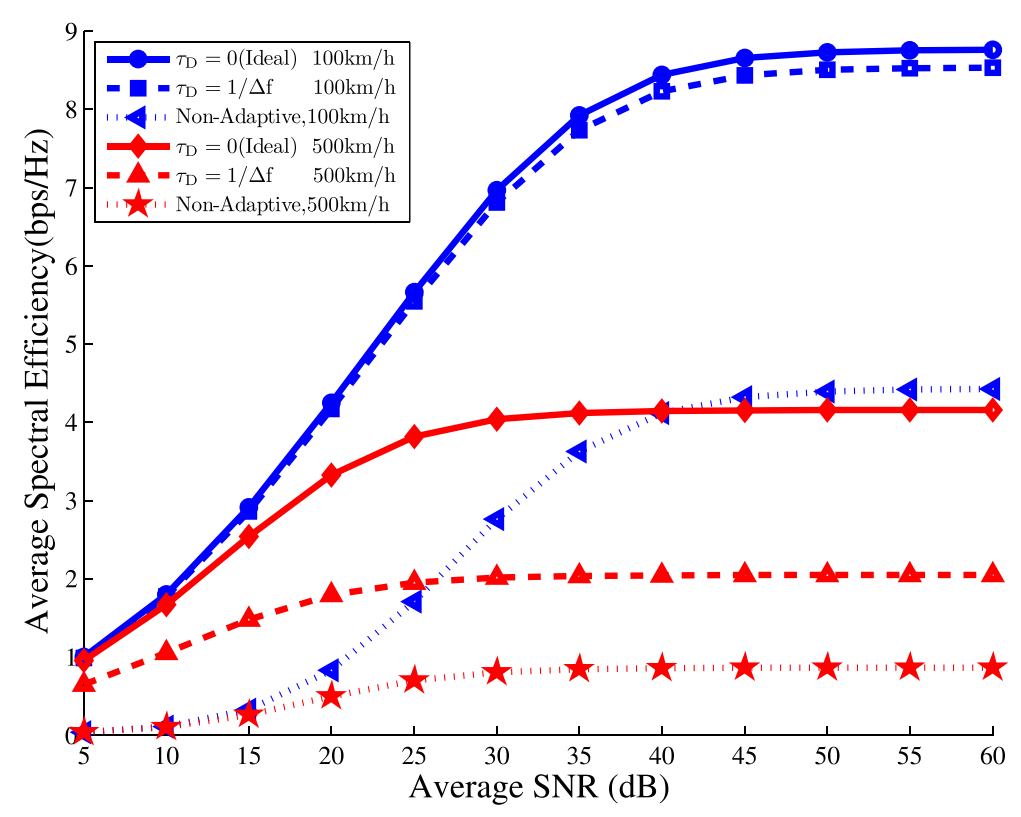

Figure 9 Average spectral efficiency for adaptive OFDM with delay CSI for different velocities. 


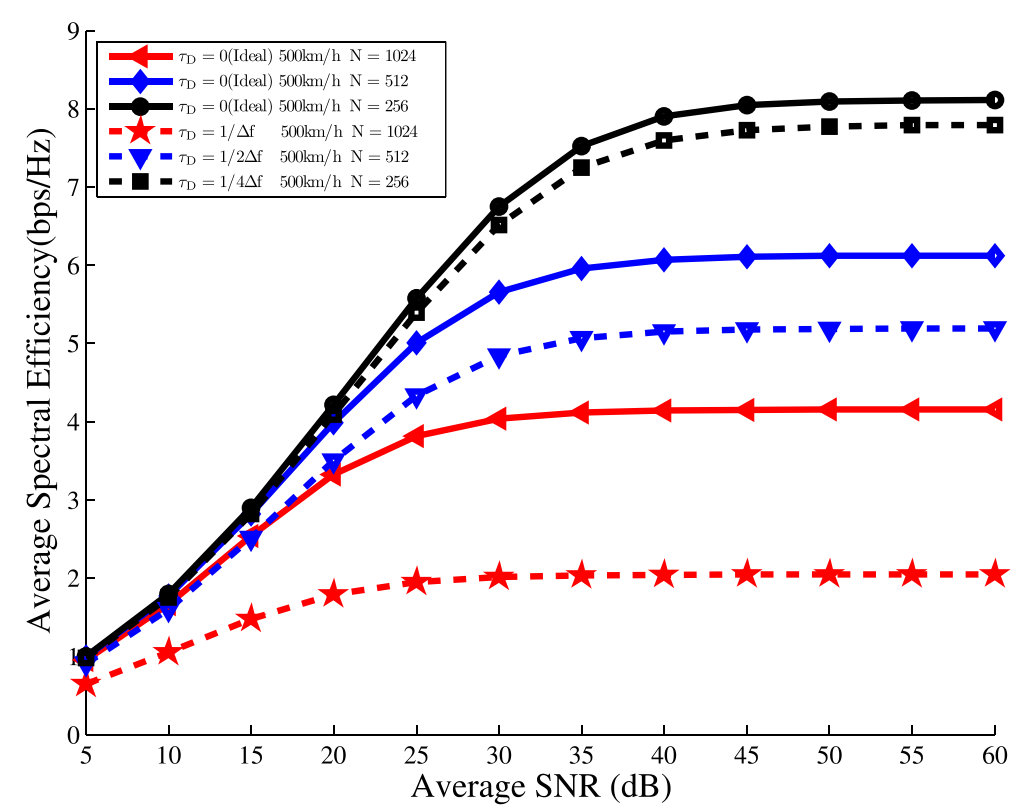

Figure 10 Average spectral efficiency for adaptive OFDM with different subcarrier bandwidth for velocity equals to $500 \mathrm{~km} / \mathrm{h}$.

Considering a BER constraint for every subcarrier, a lower bound was derived and closed form expressions were obtained for the average spectral efficiency of adaptive OFDM systems under fast fading channel with perfect and imperfect CSI. We concluded that the spectral efficiency of the proposed adaptive system under fast fading channels tends to have an average floor depending on the ICI and the average SNR. On the other hand, we observed that under imperfect CSI, the proposed adaptive system has an average floor for the spectral efficiency, depending on the imperfection in the CSI. Since very rapidly time varying channels lead to larger ICI, we have shown that the average spectral efficiency was seriously degraded under high mobility.

We have concluded from the theoretical results and the computer simulations that adaptive OFDM systems in fast fading channel have considerable gain over non-adaptive counterparts as a function of the channel estimation errors. Therefore, more accurate CSI has yielded better performance. Otherwise, the performance of the adaptive system would have degraded substantially and become worse than that of a non-adaptive system.

For adaptive OFDM system, high mobility causes inevitable delay between the channel estimation and the actual transmission at the transmitter. One of the important conclusions of this work was that increasing the spacing between the OFDM subcarriers mitigates the impact of delay on the spectral efficiency. Theoretical and numerical results have indicated that the impact of delay on the spectral efficiency of the system would be mitigated by increasing the spacing between the OFDM subcarriers, when the CP is neglected. Consequently, increasing the subcarrier bandwidth would decrease the duration of the OFDM symbol. Consequently, the time selectivity of the channel would be decreased as the frequency selectivity increasing. There exists an optimal subcarrier bandwidth when the $\mathrm{CP}$ is constant. Our future work is to find the optimal subcarrier bandwidth under some constraints.

\section{Appendices}

\section{Appendix 1: Derivation of (24)}

$\mathrm{Cap}_{\max }=\int_{\gamma 0}^{\infty} \log _{2}\left[\frac{\left(-C_{2} / \ln \left(\varepsilon / C_{1}\right)\right) \gamma[k]}{(\lambda \ln (2) \bar{S})\left(s_{\max } P_{N} \bar{\gamma}+1\right)}\right] p_{\gamma[k]}(\gamma[k]) d \gamma[k]$

Assume $\xi=-C_{2} / \ln \left(\varepsilon / C_{1}\right), \eta=\lambda \ln (2) \bar{S}, \psi=s_{\max } P_{N} \bar{\gamma}+1$.

$$
\begin{aligned}
\text { Capmax }_{\max } & =\int_{\gamma 0}^{\infty} \log _{2}\left[\frac{\xi \gamma[k]}{\eta \psi}\right] p_{\gamma[k]}(\gamma[k]) d \gamma[k] \\
& =\frac{1}{\rho_{0} \bar{\gamma}} \int_{\gamma 0}^{\infty} \log _{2}\left[\frac{\xi \gamma[k]}{\eta \psi}\right] \exp \left(-\gamma[k] / \rho_{0} \bar{\gamma}\right) d \gamma[k]
\end{aligned}
$$

For $t=\gamma[k]-\gamma_{0}$, we can express the above integral as follows

$$
\mathrm{Cap}_{\max }=\frac{1}{\ln (2) \rho_{0} \bar{\gamma}} \int_{0}^{\infty} \ln \left[\frac{\xi\left(t+\gamma_{0}\right)}{\eta \psi}\right] \exp \left(-\left(t+\gamma_{0}\right) / \rho_{0} \bar{\gamma}\right) d t
$$

By means of the integral formula [29]

$$
\int_{0}^{\infty} \ln (\chi t+1) \exp (-u t) d t=-\frac{1}{u} \exp \left(\frac{u}{\chi}\right) E i\left(-\frac{u}{\chi}\right)
$$


We obtain the final result as follows

$$
\mathrm{Cap}_{\max }=-\frac{1}{\ln (2)} E i\left(-\frac{\gamma_{0}}{\rho_{0} \bar{\gamma}}\right)
$$

Closed form expressions of (40) and (45) can be obtained by a similar approach.

\section{Appendix 2: Derivation of (28)}

Suppose the estimated channel $H_{k}^{\prime}(n)$ is the only known information about the current CSI for the $k$ th subcarrier. We will obtain the ICI power based on $H_{k}^{\prime}(n)$.

$$
\begin{aligned}
E\left\{\left|I_{k}^{\prime}\right|^{2}\right\}= & \frac{1}{N^{2}} \sum_{m=0, m \neq k}^{N-1} E\left\{\left|d_{m}\right|^{2}\right\} \sum_{n_{1}=0}^{N-1} \sum_{n_{2}=0}^{N-1} E\left\{H_{m}^{\prime}\left(n_{1}\right)\right. \\
& \left.\times H^{\prime}{ }_{m}\left(n_{2}\right)\right\} \exp \left[\frac{j 2 \pi\left(n_{1}-n_{2}\right)(m-k)}{N}\right] \\
= & \frac{1}{N^{2}} \sum_{m=0, m \neq k}^{N-1} E\left\{\left|d_{m}\right|^{2}\right\} \sum_{n_{1}=0}^{N-1} \sum_{n_{2}=0}^{N-1} E\left\{\left(H_{m}\left(n_{1}\right)\right.\right. \\
& \left.\left.+\varepsilon_{m}\left(n_{1}\right)\right)\left(H_{m}\left(n_{2}\right)+\varepsilon_{m}\left(n_{2}\right)\right)^{*}\right\} \\
& \times \exp \left[\frac{j 2 \pi\left(n_{1}-n_{2}\right)(m-k)}{N}\right] \\
= & \frac{1}{N^{2}} \sum_{m=0, m \neq k}^{N-1} E\left\{\left|d_{m}\right|^{2}\right\} \sum_{n_{1}=0}^{N-1} \sum_{n_{2}=0}^{N-1} E\left\{H_{m}\left(n_{1}\right)\right. \\
& \left.\times H_{m}\left(n_{2}\right)\right\} \exp \left[\frac{j 2 \pi\left(n_{1}-n_{2}\right)(m-k)}{N}\right] \\
& +\frac{1}{N^{2}} \sum_{m=0, m \neq k}^{N-1} E\left\{\left|d_{m}\right|^{2}\right\} N \sigma_{\varepsilon}^{2}=E_{s} P_{N} \\
& \\
& \\
& \\
&
\end{aligned}
$$

where $E_{s}=E\left\{\left|d_{m}\right|^{2}\right\}, P_{N}=\left(1-\frac{1}{N^{2}}\left(N+2 \sum_{n=1}^{N-1}(N-n)\right.\right.$ $\left.\times J_{0}\left(\frac{2 \pi f_{\max } T_{\mathrm{OFDM}} n}{N}\right)\right)$.

We obtain the instantaneous BER of each subchannel under imperfect CSI as follows

$$
\Omega^{\prime}([k]) \leq C_{1} \exp \left[\frac{-C_{2} \gamma[k] \frac{s\left(\gamma^{\prime}[k]\right)}{\bar{s}}}{\left(2^{\beta\left(\gamma^{\prime}[k]\right)}-1\right) \phi}\right]
$$

where

$$
\phi=s_{\max }^{\prime} P_{N} \bar{\gamma}+1+s_{\max }^{\prime} \bar{\gamma}(N-1) \sigma_{\varepsilon}^{2} / N
$$

\section{Competing interests}

The authors declare that they have no competing interests.

\section{Acknowledgements}

This work was supported by the National Basic Research Program of China (973 Program No. 2012CB316100), the National Science Foundation of China (NSFC, No. 61032002), and the 111 Project (No. 111-2-14). The authors would like thank the anonymous reviewers of this work whose valuable comments and corrections improved the quality of the article substantially. The authors would also like thank Weixi Zhou, Jing Zhao and Meng Chen for their constructive discussions and suggestions that very much improved this article.

\section{Author details}

${ }^{1}$ Institute of Mobile Communications, Southwest Jiaotong University, Chengdu, 610031, China. ${ }^{2}$ School of Engineering, Tibet University, Lhasa, 850000, China. ${ }^{3}$ Department of Electronics Engineering, Kadir Has University, Istanbul, 34230, Turkey. ${ }^{4}$ Institute for Space Applications and Remote Sensing, National Observatory of Athens, Athens, 15236, Greece.

Received: 15 February 2012 Accepted: 25 May 2012 Published: 2 July 2012

\section{References}

1. R Prasad, OFDM for Wireless Communications Systems (Artech House Publishers, London, 2004)

2. $\mathrm{HCWu}$, Analysis and characterization of intercarrier and interblock interferences for wireless mobile ofdm systems. IEEE Trans. Broadcast. 52(2), 203-210 (2006)

3. E Chiavaccini, GM Vitetta, Error performance of ofdm signaling over doubly-selective rayleigh fading channels. IEEE Commun. Lett. 4(11), 328-330 (2000)

4. YH Kim, I Song, HG Kim, T Chang, HM Kim, Performance analysis of a coded ofdm system in time-varying multipath rayleigh fading channels. IEEE Trans. Veh. Technol. 48(5), 1610-1615 (1999)

5. L Wan, VK Dubey, Ber performance of ofdm system over frequency nonselective fast ricean fading channels. IEEE Commun. Lett. 5(1), 19-21 (2001)

6. A Scaglione, S Barbarossa, GB Giannakis, Filterbank transceivers optimizing information rate in block transmissions over dispersive channels. IEEE Trans. Information. Theory. 45(3), 1019-1032 (1999)

7. C Liu, D Bielefeld, R Mathar, in IEEE 19th International Symposium on Personal, Indoor and Mobile Radio Communications. Optimal rate and power allocation for ofdm in the presence of channel uncertainty (IEEE, Cannes, France, 2008), pp. 1-5

8. Z Mao, X Wang, Efficient optimal and suboptimal radio resource allocation in ofdma system. IEEE Trans. Wirel. Commun. 7(2), 440-445 (2008)

9. J Huang, VG Subramanian, R Agrawal, RA Berry, Downlink scheduling and resource allocation for ofdm systems. IEEE Trans. Wirel. Commun. 8(1), 288-296 (2009)

10. SYe, RS Blum, LJ Cimini, Adaptive ofdm systems with imperfect channel state information. IEEE Trans. Wirel. Commun. 5(11), 3255-3265 (2006)

11. H Steendam, M Moeneclaey, Analysis and optimization of the performance of ofdm on frequency-selective time-selective fading channels. IEEE Trans. Commun. 47(12), 1811-1819 (1999)

12. T Keller, L Hanzo, Adaptive multicarrier modulation: a convenient framework for time-frequency processing in wireless communications. Proc. IEEE. 88(5), 611-640 (2000)

13. A Czylwik, in Global Telecommunications Conference Communications: The Key to Global Prosperity, vol. 1. Adaptive ofdm for wideband radio channels (IEEE, London, UK, 1996), pp. 713-718

14. ST Chung, A Goldsmith, in Conference Record of the Thirty-Fourth Asilomar Conference on Signals, Systems and Computers, vol. 2. Adaptive multicarrier modulation for wireless systems (IEEE, Pacific Grove, USA, 2000), pp. 1603-1607

15. H Cheon, D Hong, Effect of channel estimation error in ofdm-based wlan. IEEE Commun. Lett. 6(5), 190-192 (2002) 
16. L Rugini, P Banelli, Ber of ofdm systems impaired by carrier frequency offset in multipath fading channels. IEEE Trans. Wirel. Commun. 4(5), 2279-2288 (2005)

17. K Nehra, M Shikh-Bahaei, Spectral efficiency of adaptive mqam/ofdm systems with cfo over fading channels. IEEE Trans. Veh. Technol. 60(3), 1240-1247 (2011)

18. SS Das, E De Carvalho, R Prasad, Performance analysis of ofdm systems with adaptive sub carrier bandwidth. IEEE Trans. Wirel. Commun. 7(4), 1117-1122 (2008)

19. JH Wen, CH Chiang, TJ Hsu, HL Hung, Resource management techniques for ofdm systems with the presence of inter-carrier interference. Wirel. Personal Commun, 1-21 (2011). doi:10.1007/s11277-011-0270-2

20. X Cai, GB Giannakis, performance and suppressing intercarrier interference in wireless mobile ofdm. IEEE Trans. Commun. 51(12), 2047-2056 (2003)

21. $Y L i, L J$ Cimini Jr., Bounds on the interchannel interference of ofdm in time-varying impairments. IEEE Trans. Commun. 49(3), 401-404 (2001)

22. P Robertson, $\mathrm{S}$ Kaiser, Analysis of doppler spread perturbations in ofdm (a) systems. Eur. Trans. Telecommun. 11(6), 585-592 (2000)

23. J Li, M Kavehrad, Effects of time selective multipath fading on ofdm systems for broadband mobile applications. IEEE Commun. Lett. 3(12), 332-334 (1999)

24. B Stantchev, G Fettweis, Time-variant distortions in ofdm. IEEE Commun. Lett. 4(10), 312-314 (2000)

25. M Russell, GL Stuber, in IEEE 45th Vehicular Technology Conference, vol. 2. Interchannel interference analysis of ofdm in a mobile environment (IEEE, Chicago, USA, 1995), pp. 820-824

26. YS Choi, PJ Voltz, FA Cassara, On channel estimation and detection for multicarrier signals in fast and selective rayleigh fading channels. IEEE Trans. Commun. 49(8), 1375-1387 (2001)

27. E Panayirci, H Senol, HV Poor, Joint channel estimation, equalization, and data detection for ofdm systems in the presence of very high mobility. IEEE Trans. Signal Process. 58(8), 4225-4238 (2010)

28. ST Chung, AJ Goldsmith, Degrees of freedom in adaptive modulation: a unified view. IEEE Trans. Commun. 49(9), 1561-1571 (2001)

29. IS Gradshtein, IM Ryzhik, Table of Integrals, Series and Products, 7th edn. (Academic Press, New York, 2007)

30. E Panayirci, H Dogan, V Poor, Low-complexity map-based successive data detection for coded ofdm systems over highly mobile wireless channels. IEEE Trans. Veh. Technol. 60(6), 2849-2857 (2011)

31. A Olfat, M Shikh-Bahaei, Optimum power and rate adaptation for MQAM in Rayleigh flat fading with imperfect channel estimation. IEEE Trans. Veh. Technol. 57(4), 2622-2627 (2008)

doi:10.1186/1687-1499-2012-208

Cite this article as: Dong et al:: Effect of power and rate adaptation on the spectral efficiency of MQAM/OFDM system under very fast fading channels. EURASIP Journal on Wireless Communications and Networking 2012 2012:208.

\section{Submit your manuscript to a SpringerOpen ${ }^{\odot}$ journal and benefit from:}

- Convenient online submission

- Rigorous peer review

- Immediate publication on acceptance

- Open access: articles freely available online

- High visibility within the field

- Retaining the copyright to your article

Submit your next manuscript at $\boldsymbol{\wedge}$ springeropen.com 